\title{
Effect of Hyssopus officinalis L. on inhibiting airway inflammation and immune regulation in a chronic asthmatic mouse model
}

\author{
XIAOJUAN MA ${ }^{1,2^{*}}$, XIUMIN MA ${ }^{3 *}$, ZHIXING MA ${ }^{4 *}$, JING WANG $^{1,2^{*}}$, \\ ZHAN SUN ${ }^{2}$, WENYAN YU ${ }^{2}$, FENGSEN LI ${ }^{1}$ and JIANBING DING ${ }^{2}$ \\ ${ }^{1}$ Xinjiang National Clinical Research Base of Traditional Chinese Medicine, Xinjiang Medical University, \\ Ürümqi, Xinjiang 830011; ${ }^{2}$ Department of Immunology, College of Basic Medicine, Ürümqi, Xinjiang 830011; \\ ${ }^{3}$ Department of Clinical Laboratory, First Affiliated Hospital of Xinjiang Medical University, Ürümqi, Xinjiang 830054; \\ ${ }^{4}$ Ürümqi General Hospital of Lanzhou Military Area Command, Ürümqi, Xinjiang 830000, P.R. China
}

Received February 14, 2014; Accepted July 14, 2014

DOI: 10.3892/etm.2014.1978

\begin{abstract}
The Uygur herb, Hyssopus officinalis L., has been demonstrated to affect the levels of a number of cytokines in asthmatic mice, including interleukin-4, -6 and -17 and interferon- $\gamma$. In the present study, the effect of Hyssopus officinalis $\mathrm{L}$. on airway immune regulation and airway inflammation was investigated in a mouse model of chronic asthma. A total of $32 \mathrm{BALB} / \mathrm{c}$ mice were randomly divided into four groups, which included the normal, chronic asthmatic, dexamethasone treatment and Hyssopus officinalis L. treatment groups. Mice were sensitized and challenged with ovalbumin to establish an asthma model and the ratio of eosinophils (EOS) in the bronchoalveolar lavage fluid (BALF) was determined. In addition, the levels of immunoglobulin (Ig)E and $\mathrm{IgG}$ were detected using an enzyme-linked immunosorbent assay. The degree of airway mucus secretion was observed using the periodic acid-Schiff stain method. The results demonstrated that the ratio of EOS in the BALF and the level of serum IgE in the chronic asthmatic and dexamethasone treatment groups increased, while the level of serum IgG decreased, when compared with the normal group. In addition, excessive secretion of airway mucus was observed in these two groups. However, the EOS ratio in the BALF and the levels of serum $\mathrm{IgE}$ and $\mathrm{IgG}$ in the Hyssopus officinalis $\mathrm{L}$. treatment group
\end{abstract}

Correspondence to: Mr. Fengsen Li, Xinjiang National Clinical Research Base of Traditional Chinese Medicine, Xinjiang Medical University, 116 Huanghe Road, Ürümqi, Xinjiang 830011, P.R. China E-mail: 528974942@qq.com

Mrs. Jianbing Ding, Department of Immunology, College of Basic Medicine, Xinjiang Medical University, 393 Xinyi Road, Ürümqi, Xinjiang 830011, P.R. China

E-mail: 1601379937@qq.com

*Contributed equally

Key words: chronic asthma, Hyssopus officinalis L., airway inflammation, immune regulation were similar to the results observed in the normal group. In conclusion, Hyssopus officinalis L. not only plays an anti-inflammatory role by inhibiting the invasion of EOS and decreasing the levels of IgE, but also affects immune regulation.

\section{Introduction}

Bronchial asthma is a common clinical disease. An epidemiological study found that the rates of morbidity, mortality and prevalence of bronchial asthma are increasing worldwide. In China, the incidence of bronchial asthma has doubled over the past decade. The World Health Organization defines bronchial asthma as a chronic airway inflammation that is caused by various inflammatory cells, including eosinophils (EOS), mast cells and $\mathrm{T}$ lymphocytes (1-3).

Chronic airway inflammation is characterized by the infiltration of EOS, an increase in serum immunoglobulin (Ig)E and excessive secretion of airway mucus (4-6), which result in airway structural changes and may even develop into refractory asthma or severe asthma (7). Hyssopus officinalis L., an Uygur medicine, is a perennial herb of Labiatae. The herb has been shown to relieve coughing and asthma; however, the underlying mechanisms are yet to be elucidated. Previous studies have shown that Hyssopus officinalis L. plays an anti-inflammatory role by regulating the secretion of interleukin (IL)-4, IL-17 and interferon- $\gamma$ (IFN- $\gamma$ ), as well as regulating the imbalance between Th1/Th2 cytokines (8-10). However, the role of Hyssopus officinalis L. in the regulation of immune function has not yet been investigated. Therefore, the aim of the present study was to investigate the effect of Hyssopus officinalis L. on immunity in a mouse model of chronic asthma. In addition, the study investigated whether Hyssopus officinalis $\mathrm{L}$. was able to increase immune reactions and thus, may be used to improve and perfect the mechanisms underlying the treatment of asthma.

\section{Materials and methods}

Extract preparation. Crude herbs (200 g) were extracted using $6,000 \mathrm{ml}$ water. The solvents in the extracts were removed via 
rotary evaporation under reduced pressure (EYELA-digital water bath SB-100; Eyela, Tokyo, Japan). The aqueous extract was then freeze-dried and stored at $4^{\circ} \mathrm{C}$. The aqueous extract was diluted with saline water prior to use. In a previous study, the toxicology and dosage were assessed (11).

Animals and model establishment. A total of 32 female $\mathrm{BALB} / \mathrm{c}$ mice were purchased from the Animal Experiment Center at Xinjiang Medical University (Ürümqi, China). The mice were housed in microisolator cages and received food and water. The laboratory temperature was maintained at $24 \pm 1^{\circ} \mathrm{C}$, and the relative humidity was maintained between 40 and $80 \%$. All the experimental protocols were approved by the regional Animal Ethics Committee of Xinjiang Medical University.

The BALB/c mice (age, 6-8 weeks) were randomly divided into four groups, which included the normal, chronic asthma, dexamethasone and Hyssopus officinalis L. groups. The chronic asthma, dexamethasone and Hyssopus officinalis $\mathrm{L}$. groups were administered an intraperitoneal injection of $0.2 \mathrm{ml}$ sensitizing agent, which contained $100 \mu \mathrm{g}$ ovalbumin (OVA; Sigma-Aldrich, St. Louis, MO, USA) and $1 \mathrm{mg}$ aluminum hydroxide gel, on days 1 and 15 . In addition, initiating on day 22 , the mice were administered $1 \%$ OVA for 30 min three times a week for eight weeks. The normal group were treated with phosphate-buffered saline (PBS) instead of OVA (12). Drugs were chronically administered to the animals $1 \mathrm{~h}$ prior to the challenges $[0.005 \mathrm{mg} / 10 \mathrm{~g}$ body weight dexamethasone (TianYaoYaoYe Co., Ltd. Hubei, China); 0.04 g/10 g body weight Hyssopus officinalis L. (Pharmacy of Uyghur Hospital, Xinjiang, China)], once per day for eight weeks. Animals were euthanized following the last challenge and samples were collected.

Bronchoalveolar lavage fluid (BALF) and serum harvesting. The chest was opened and the bronchus principalis dexter was ligated in the bifurcation. BALF was collected using $0.4 \mathrm{ml}$ PBS, three times in total according to a previously described method $(13,14,15)$. The number of neutrophils was determined using the CytoSpin ${ }^{\mathrm{TM}}$ centrifuge (Thermo Fisher Scientific, Waltham, MA, USA).

The levels of serum $\operatorname{IgE}$ and $\operatorname{IgG}$ were analyzed using an ELISA, measuring the change of absorbance at $450 \mathrm{~nm}$ (Bei Lai Yin Biological Technology Co., Ltd., Wuhan, China).

Periodic acid-Schiff (PAS) staining and analysis. Lung tissue samples were stained with PAS (Jiancheng Bioengineering Institute of Nanjing, Nanjing, China) and analyzed using an optical microscope (Olympus CX22, Olympus Tokyo, Japan). A minimum of ten airways were observed in each section. The PAS positively-stained cells were counted and scored using previously described methods $(16,17,18)$. The scoring method used was as follows: $0,<5 \% ; 1,5-25 \% ; 2,25-50 \% ; 3,50-5 \%$; and $4,>75 \%$ PAS positively-stained cells.

Statistical analysis. SPSS 17.0 statistical software (SPSS, Inc., Chicago, IL, USA) was used for statistical analysis. Data are presented as the mean \pm standard error of the mean. Comparisons between the groups were performed using analysis of variance followed by the Dunnett's test, where

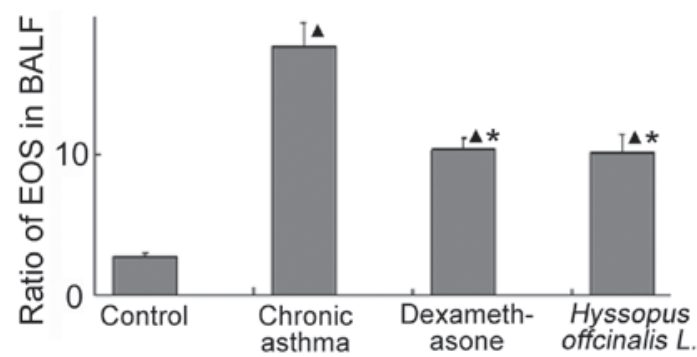

Figure 1. Percentage of EOS to white blood cells in the BALF. Data are presented as the mean \pm standard error of the mean. ${ }^{\Delta} \mathrm{P}<0.05$, vs. normal group; ${ }^{\star} \mathrm{P}<0.05$, vs. chronic asthma group. BALF, bronchoalveolar lavage fluid; EOS, eosinophils

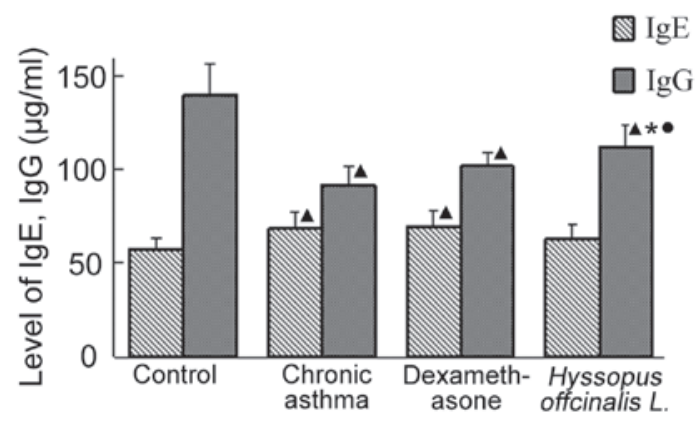

Figure 2. Comparison of the serum IgE and $\operatorname{IgG}$ concentration among the groups. Data are presented as the mean \pm standard error of the mean. ${ }^{\Delta} \mathrm{P}<0.05$, vs. normal group; ${ }^{\star} \mathrm{P}<0.05$, vs. chronic asthma group; ${ }^{\bullet} \mathrm{P}<0.05$, vs. dexamethasone group. Ig, immunoglobulin.

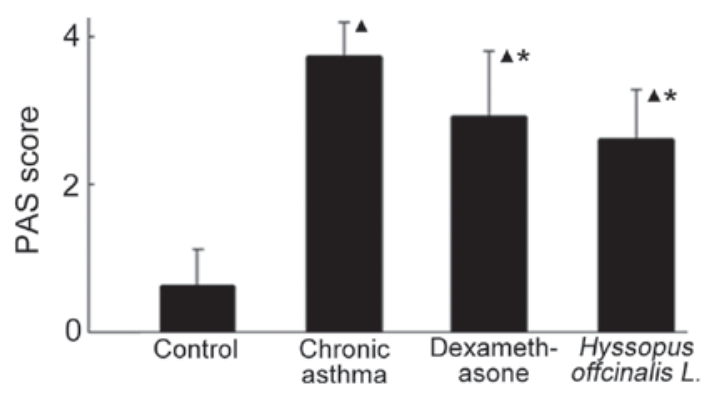

Figure 3. Comparison of airway mucus secretion among the groups. Data are presented as the mean \pm standard error of the mean. ${ }^{\boldsymbol{\Delta}} \mathrm{P}<0.05$, vs. normal group; ${ }^{\star} \mathrm{P}<0.05$, vs. chronic asthma group. PAS, periodic acid-Schiff.

$\mathrm{P}<0.05$ was considered to indicate a statistically significant difference.

\section{Results}

Behavioral alterations. Following the challenge with OVA, the mice presented with a number of symptoms, including anxiety, nose scratching, coughing, inspiratory dyspnea, shortness of breath, retardation, piloerection and cyanosis. The activity decreased following continuous challenges. However, the symptoms were relieved in the dexamethasone and Hyssopus officinalis L. groups.

Ratio of EOS in the BALF. Fig. 1 shows that the percentage of EOS in the chronic asthma group was higher compared 
A

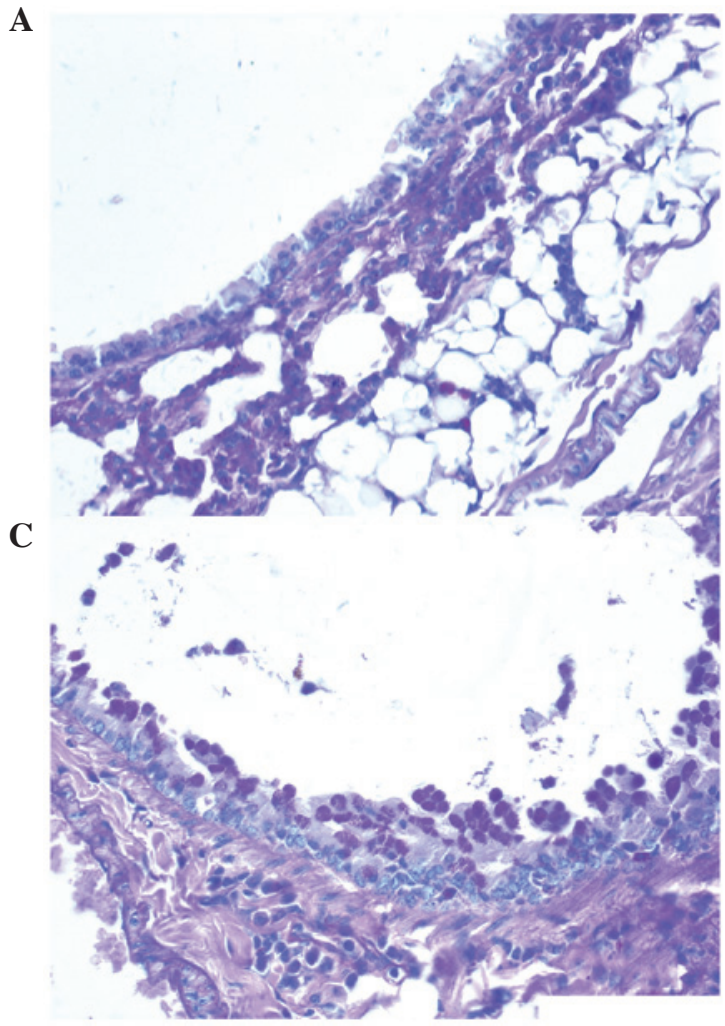

B

D

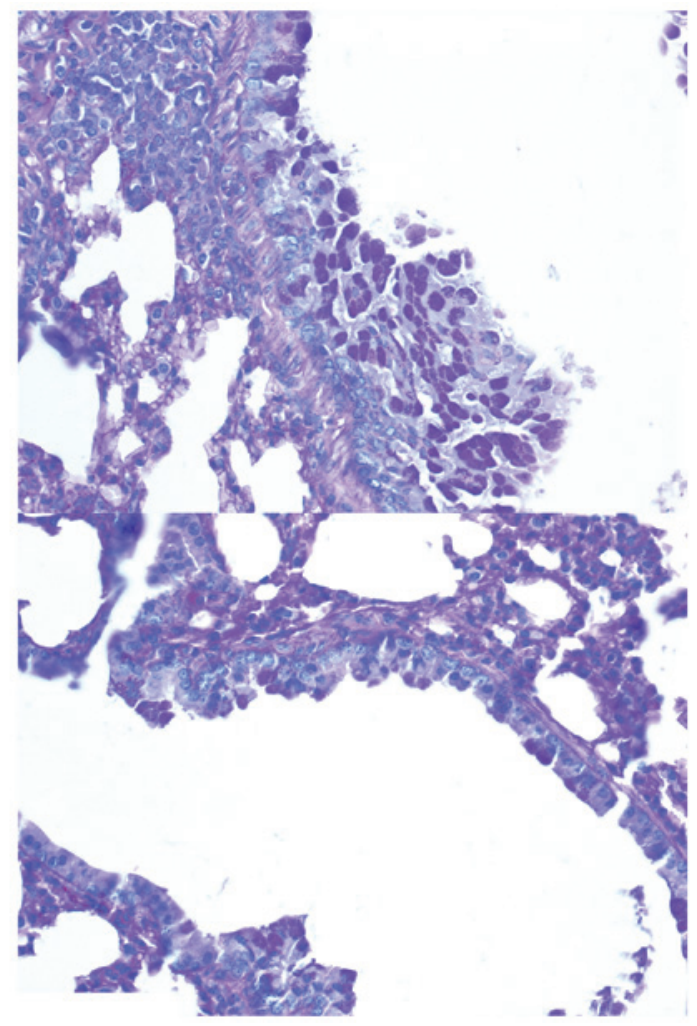

Figure 4. Lung tissue staining by PAS was used to observe the secretion of airway mucus in the (A) normal, (B) chronic asthma, (C) dexamethasone and (D) Hyssopus offcinalis L. groups (magnification, $\mathrm{x} 40$ ). Analysis shows the neutral mucus as purple in the alveolar space and the nucleus as violet. PAS, periodic acid-Schiff.

with the other groups. Furthermore, the ratio of EOS in the dexamethasone group was similar to the ratio observed in the Hyssopus officinalis L. group (10.27 \pm 0.85 and $10.08 \pm 1.29$, respectively).

Level of IgE and IgG in the serum. As shown in Fig. 2, the level of IgE in the Hyssopus officinalis L. group were similar to the levels observed in the normal group. In addition, the level of $\operatorname{IgG}$ in the chronic asthma and dexamethasone groups were lower compared with the normal group; however, the concentration of $\operatorname{IgG}$ in the Hyssopus officinalis L. group was higher compared with the chronic asthma and dexamethasone groups.

Secretion of mucus in the airway. The secretion of airway mucus in the chronic asthma group was aggravated (Figs. 3 and 4). In addition, the secretion of mucus increased in the dexamethasone and Hyssopus officinalis L. groups; however, the levels were highest in the chronic asthma group.

\section{Discussion}

Asthma is a type I allergic disease, and IgE has an important role in the development of asthma (19). Previous studies have demonstrated that there are various levels of inflammatory responses in patients who suffer from asthma, and allergic airway inflammation with increasing EOS is the main pathological feature of asthma $(20,21)$. Numerous studies have identified a correlation between the infiltrate level of EOS and the severity of airway inflammation (22-24). The variety of cell types present in BALF may reflect the degree of inflammation in the peripheral airways, which is also the main factor causing bronchial hyperresponsiveness in asthma (25). Therefore, airway inflammation is the basic condition of reversible airway inhibition, as well as non-special hyperresponsiveness of the bronchus. In the present study, the percentage of EOS in the BALF and the levels of IgE in the chronic asthma group were higher compared with the normal group $(\mathrm{P}<0.05)$. PAS staining revealed that mucus secretion was elevated in the chronic asthma group. Furthermore, the level of IgG was observed to decrease in the chronic asthma group. These results indicated that the immune reaction in individuals suffering chronic asthma is restrained.

Hormonal nebulizer inhalation has become a common clinical treatment for non-special airway inflammation; however, this suppresses the immune system. Due to the geography and climate of the Autonomous region of Xinjiang, the incidence of asthma is increasing. There are a number of effective strategies and medicines for treating asthma in Uygur medicine. Hyssopus officinalis L. is an Uygur medicine used for the treatment of a number of conditions, including asthma, coughing, fever and rheumatism (26). In a number of previous studies, Hyssopus officinalis $\mathrm{L}$. has been demonstrated to affect the expression of a number of cytokines (8-10). In the present study, mice treated with Hyssopus officinalis L. and dexamethasone were shown to have decreased levels of EOS in the BALF when compared with mice in the chronic asthma group $(10.08 \pm 1.29$, $10.27 \pm 0.85$ and $17.56 \pm 1.71$, respectively; $\mathrm{P}<0.05$ ). In addition, the level of IgE and the secretion of airway mucus were reduced. Mice treated with Hyssopus officinalis L. were also shown 
to have higher levels of $\mathrm{IgG}$ when compared with the dexamethasone and asthma groups $(111.71 \pm 11.79,101.69 \pm 7.02$ and $91.27 \pm 10.69$, respectively; $\mathrm{P}<0.05)$. These observations indicate that Hyssopus officinalis L. may exhibit an anti-inflammatory effect by inhibiting the infiltration of EOS and reducing the level of $\mathrm{IgE}$ in the lung tissue; thus, regulating immunity.

In conclusion, the results of the present study demonstrate the effect of Hyssopus officinalis L. on chronic asthma, and may provide a novel therapeutic strategy for the treatment of chronic asthma. However, further investigation is required to determine the specific mechanism.

\section{Acknowledgements}

The study was supported by grants for innovation from Xinjiang Medical University (no. XJC2012-20), the Autonomous Region of Xinjiang Leading Academic Discipline Project of Pathogeny Biology (no. XYDXK 50780328) and the National Science Foundation of the Autonomous Region of Xinjiang (no. 200821129).

\section{References}

1. Lemanske RF Jr and Busse WW: Asthma: clinical expression and molecular mechanisms. J Allergy Clin Immunol 125 (Suppl 2): S95-S102, 2010.

2. Herrick CA and Bottomly K: To respond or not to respond: T cells in allergic asthma. Nat Rev Immunol 3: 405-412, 2003.

3. Wei M, Chu X, Guan M, et al: Protocatechuicacid suppresses ovalbumin-induced airway inflammation in a mouse allergic asthma model. Int Immunopharmacol 4: 780-788, 2013.

4. Wang W, Huang KW, Wu BM, Wang YJ and Wang C: Correlation of eosinophil counts in induced sputum and fractional concentration of exhaled nitric oxide and lung functions in patients with mild to moderate asthma. Chin Med J (Engl) 125: 3157-3160, 2012.

5. Hannah JG and Sutton BJ: IgE in allergy and asthma today. Nat Rev Immunol 8: 205-217, 2008.

6. Rogers DF: Airway mucus hypersecretion in asthma: an undervalued pathology? Curr Opin Pharmacol 4: 241-250, 2004.

7. Zhao KZ, Lu JR, Zhang YF, et al: Changes of the levels of interleukin-13 and total immunoglobulin $\mathrm{E}$ in children with asthma. Jilin Da Xue Xue Bao. Yi Xue Ban 29: 212-213, 2003 (In Chinese)

8. Wang YN, Ma J, Ma XM, et al: Effects of Uygur herb Hyssopus officinalis L. on cytokines in allergic asthma mice. Shanghai Zhong Yiyao Daxue Xuebao 22: 58-60, 2008.

9. Hou M, Zhu M, Ma XM, et al: Effects of Uygur medicine Hyssopus officinalis L. on serum IL-17 level and balance of Th1/Th2 of asthma rats. Zhongguo Xian Dai Yi Xue Za Zhi 20: 365-368, 2010 (In Chinese).
10. Hou M, Ma XM, Ding JB, et al: Effect of Uygur medicine Hyssopus officinalis L. on serum eotaxin-2, eotaxin-3 and sP-selectin level of asthma rats. Science \& Technology Review 28: 90-93, 2009

11. Ma XP, Ma XM, Ding JB, et al: Extraction process of polysaccharide from Hyssopus officinalis. Chin JMAP 28: 437-439, 2011.

12. Du Q, Zhang Q, Shen L, et al: Effects of astragaloside IV on airway remodeling in a murine model of chronic asthma. Zhongguo Yao Li Xue Tong Bao 27: 1430-1434, 2011 (In Chinese).

13. Kim MS, Cho KA, Cho YJ and Woo SY: Effects of interleukin-9 blockade on chronic airway inflammation in murine asthma models. Allergy Asthma Immunol Res 5: 197-206, 2013.

14. Chong L, Zhang W, Nie Y, Yu G, Liu L, et al: Protective effect of curcumin on acute airway inflammation of allergic asthma in mice through Notch1-GATA3 signaling pathway. Inflammation Apr 6, 2014 (Epub ahead of print).

15. Blonder JP, Mutka SC, Sun X, Qiu J, et al: Pharmacologic inhibition of S-nitrosoglutathione reductase protects against experimental asthma in BALB/c mice through attenuation of both bronchoconstriction and inflammation. BMC Pulm Med 14: 3, 2014.

16. Asano T, Kume H, Taki F, et al: Thalidomide attenuates airway hyperresponsiveness and eosinophilic inflammation in a murine model of allergic asthma Biol Pharm Bull 33: 1028-1032, 2010.

17. Lu S, Li C, Ming M and Luo Z: Inhalation of inactivated Mycobacterium phlei down-regulates expression of nuclear factor-kappa B and intercellular adhesion molecule in asthmatic mice. Chinese J Pathophysiol 30: 333-338, 2014.

18. Zhang W, Zhu T, Wang D, et al: Rosuvastatin attenuates airway inflammation of chronic asthma in mice. Med J West China 26 : 150-153, 2014

19. Chu Y: Progress in the association of high affinity IgE receptor with asthma. Int J Pediatrics 38: 280-282, 2011.

20. Stone KD, Prussin C and Metcalfe DD: IgE, mast cells, basophils, and eosinophils. J Allergy Clin Immunol 125 (Suppl 2): S73-S80, 2010.

21. Locksley RM: Asthma and allergic inflammation. Cell 140: 777-783, 2010.

22. Hargreave FE and Nair P: Point: Is measuring sputum eosinophils useful in the management of severe asthma? Yes. Chest 139: 1270-1273, 2011.

23. Regina MCPinto, Alberto C, Luciene A, et al: Clinical characteristics and possible phenotypes of an adult severe asthma population. Respir Med 106: 47-56, 2012.

24. Jatakanon A, Lim S, Kharitonov SA, Chung KF and Barnes PJ: Correlation between exhaled nitric oxide, sputum eosinophils, and methacholine responsiveness in patients with mild asthma. Thorax 53: 91-95, 1998.

25. Ichiki H, Hoshino T, Kinoshita T, et al: Thioredoxin suppresses airway hyperresponsiveness and airway inflammation in asthma. Biochem Biophys Res Commun 334: 1141-1148, 2005.

26. Liu YM and Wushati Y (eds): Hyssopus offcinalis L.. In: J Uygur Med. Xinjiang Science \& Technology \& Hygiene Publishing House, Ürümqi, China pp423-429, 1999. 